\title{
An edge computing framework based on OFDM radar for low grazing angle target tracking
}

\author{
Vahid Karimi*, Reza Mohseni and Mohammad R. Khosravi
}

\begin{abstract}
In this paper, a novel adaptive algorithm to detect and track targets with low grazing angle is addressed. For this purpose, an orthogonal frequency division multiplexing (OFDM) radar signal is employed through an edge computing framework over the radar platform. However, detecting the targets in the low grazing angle area is a great challenge due to severe multipath reflection effects. The Earth's curvature geometry model is presented as the multipath propagation model. Based on the fact that the different scattering centers of a target resonate at different frequencies, we use the optimized OFDM waveform and propose a novel target tracking procedure for low grazing angle target tracking scenarios. The obtained results show that using an OFDM radar waveform provides a more uniform detection coverage in the presence of multipath propagation such that this will fill in the nulls. Finally, simulations are used to compare the performance of the proposed OFDM waveform with the conventional equal-power, the generalized likelihood ratio (GLR)-based and single-carrier waveforms.
\end{abstract}

Keywords: Orthogonal frequency division multiplexing signal, Low grazing angle, Target tracking, Multipath reflection

\section{Introduction}

Angle of arrival (AOA) estimation in a low grazing angle is one of the most challenging problems in radar fields [1-3], where the multipath effect is the main problem. In this case, the detection and tracking performance severely deteriorated due to the random phase variation of indirect signals. The direct and reflected returns coherently combined at the receiver with the peaks (maxima) and nulls (minima) corresponding to complete constructive and destructive interference, respectively, depending upon the phase difference. It had been shown that multipath reflections affects categorized into two different aspects, namely, multipath suppression and multipath utilization. Some methods regard the multipath effect as a clutter component and try to suppress it [4-12]. Numerous methods, such as spatial smoothing techniques, proposed removing the coherency [13, 14]. It had been demonstrated in [15] that the detection

\footnotetext{
*Correspondence: v.karimi@sutech.ac.ir

Department of Electrical and Electronic Engineering, Shiraz University of Technology, Shiraz, Iran
}

performance could be enhanced via multipath utilization with a statistical multiple-input multiple-output (MIMO) radar. The authors have further investigated a hybrid MIMO radar for detecting low grazing angle targets in multipath propagation scenarios [16].

We know that the location of the multipath nulls is frequency-dependent in range and elevation angle. Some methods have shown that using the advantage of multipath propagation, which provides spatial diversity of radar systems and frequency diversity of orthogonal frequency division multiplexing (OFDM) waveforms, and a remarkable improvement will be achieved [17]. The authors presented an adaptive waveform design method based on maximizing the Mahalanobis distance to improve the target detection performance in low grazing angle situations. We consider the OFDM signaling scheme [18-25], which is one of the ways to accomplish the simultaneous use of several subcarriers. Furthermore, the monopulse method, which has been utilized in many studies for target detection and tracking [26-31], has been applied for the angle of arrival estimation. A new adaptive beamforming 
method has been investigated in [32] for low-angle target tracking under multipath scenarios. They proposed a new monopulse technique that utilizes an iterative interference cancellation algorithm to overcome performance degradation.

In this study, we have investigated a new recursive algorithm which provides an efficient spectrum that uses optimized OFDM waveform proposed in [33]. The angle of arrival for each distance of a target is estimated based on the signal error obtained by the amplitude monopulse method. Simulation results show that the proposed approach allows to intelligently selecting subcarriers to fill in the nulls caused by the corresponding destructive effect of multipath.

Our contributions can be summarised as follows:

- Analogous to [17], a realistic multipath reflection model, is considered for the Earth's curvature, while an idealistic model had been considered in the literature [12].

- Our work is to improve the target tracking performance in a low grazing angle region by employing the optimized OFDM waveform. To do this, we have proposed a new recursive method for low grazing angle target tracking, which could fill in multipath nulls and have a good performance in low grazing angle scenarios.

The rest of this paper is organized as follows: in Section 2, the ray reflection model is described to obtain the formulas that will be used to represent the geometrical environment of the low-angle target. In Section 3, the model of OFDM measurement for an extended target case, including multipath effect, has been presented. An extended target model with multiple approximately fixed scattering centers has been considered and assumed that the target may have a bulk velocity. In Section 4, the frequency diversity of OFDM waveforms has been utilized, and a recursive low-angle target tracking method is presented to mitigate multipath nulls. Simulation results are presented in Section 5 for selected experiments to illustrate the excellent performance of the proposed target tracking algorithm for low grazing angle scenarios. Finally, we summarize and conclude the paper in Section 6.

Notation: throughout this correspondence, $E[],.(.)^{H},(.)^{T},(),. I_{M}, \operatorname{Re}(),. \operatorname{Tr}($.$) and logdet denote$ expectation operator, conjugate transpose, transpose, estimate, $M \times M$ identity matrix, real part, trace, and logarithm-determinant, respectively.

\section{Geometric background}

The spherical-earth model geometry shown in Fig. 1 is used to solve for the path length difference $\Delta R$, target elevation angle $\theta_{d}$, reflection point depression angle $\theta_{r}$. Then the grazing angle $\Phi$ given the radar antenna height $h_{a}$, target altitude $h_{t}$, and the target slant range $R_{d}$.

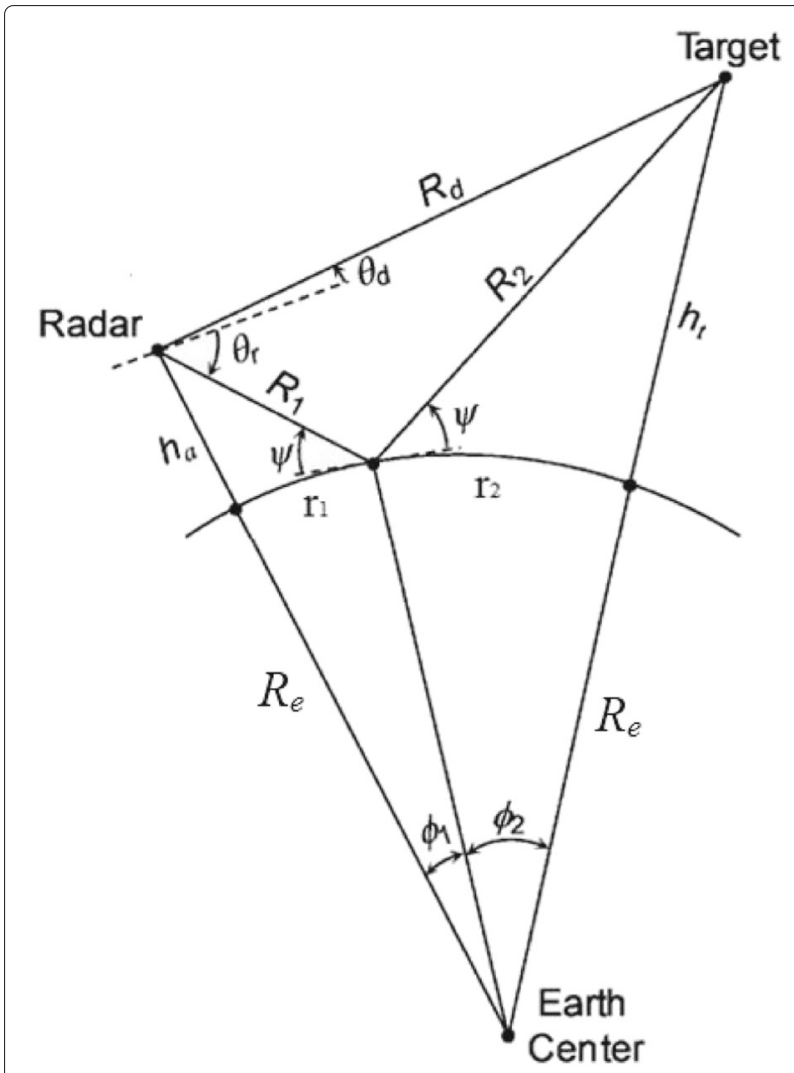

Fig. 1 Spherical-earth model geometry [17]

Firstly, obtain the effective Earth radius as [20]

$$
R_{e}=\frac{4}{3} r_{e}
$$

where $r_{e}$ stands for the actual radius of the earth. The target elevation angle $\theta_{d}$ can be derived from applying the law of cosine (LOC) as

$$
\theta_{d}=\sin ^{-1}\left(\frac{2 R_{e}\left(h_{t}-h_{a}\right)+h_{t}^{2}-h_{a}^{2}-R_{d}^{2}}{2\left(R_{e}+h_{a}\right) R_{d}}\right)
$$

The target ground range $r=r_{1}+r_{2}$ is defined as [20]

$$
r=2 R_{e} \sin ^{-1} \sqrt{\frac{R_{d}^{2}-\left(h_{t}-h_{a}\right)^{2}}{4\left(R_{e}+h_{a}\right)\left(R_{e}+h_{t}\right)}}
$$

The ground range $r_{1}$ is given by

$$
r_{1}=\frac{r}{2}-p \sin \left(\frac{\phi}{3}\right)
$$

where

$$
p=\frac{2}{\sqrt{3}} \sqrt{R_{e}\left(h_{t}+h_{a}\right)+\left(\frac{r}{2}\right)^{2}}
$$

And 


$$
\phi=\sin ^{-1}\left(\frac{2 R_{e} r\left(h_{t}-h_{a}\right)}{p^{3}}\right)
$$

Using the law of cosines, the reflected ray path length from the radar antenna to the reflection point can be computed as

$$
\begin{aligned}
& R_{1}=\sqrt{h_{a}^{2}+4 R_{e}\left(h_{a}+R_{e}\right) \sin ^{2}\left(\frac{r_{1}}{2 R_{e}}\right)} \\
& R_{2}=\sqrt{h_{t}^{2}+4 R_{e}\left(h_{t}+R_{e}\right) \sin ^{2}\left(\frac{r_{2}}{2 R_{e}}\right)}
\end{aligned}
$$

The depression angle of the reflected leg is computed as

$$
\theta_{r}=\sin ^{-1}\left(\frac{2 R_{e} h_{a}+h_{a}^{2}+R_{1}^{2}}{2\left(R_{e}+h_{a}\right) R_{1}}\right)
$$

And finally, the grazing angle at the reflection point is

$$
\psi=\sin ^{-1}\left(\frac{2 R_{e} h_{a}+h_{a}^{2}-R_{1}^{2}}{2 R_{e} R_{1}}\right)
$$

The path length difference between the direct and reflected legs can now be written as

$$
\Delta R=\left(R_{1}+R_{2}\right)-R_{d}=\frac{4 R_{1} R_{2} \sin ^{2}(\psi)}{R_{1}+R_{2}+R_{d}}
$$

\section{Preliminaries}

We consider a multicarrier OFDM signaling system consists of $L$ active subcarriers, a pulse duration of $T$ seconds, and a bandwidth of $B \mathrm{~Hz}$. The complex-valued baseband OFDM signal can be written as

$$
s(t)=\sum_{l=0}^{L-1} a_{l} \exp (j 2 \pi l \Delta f t)
$$

$\Delta f=1 / T$ represents the subcarrier spacing and $a_{l}$ denotes the complex weights transmitted over different subcarriers, satisfying $\sum_{l=0}^{L-1}\left|a_{l}\right|^{2}=1$. Now let $f_{c}$ be the carrier frequency, then the OFDM transmitted signal can be written as

$$
\tilde{s}(t)=2 \operatorname{Re}\left\{\sum_{l=0}^{L-1} a_{l} \exp \left(j 2 \pi f_{l} t\right)\right\}
$$

where

$$
f_{l}=f_{c}+l \Delta f \quad l=0,1, \ldots, L-1
$$

The complex envelope of the return signal along the $p$-th path at the terminals of the $l$ th subchannel is given by

$$
\tilde{r}_{l}(t)=x_{l p} \tilde{s_{l}}\left(\gamma_{p}\left(t-\tau_{p}\right)\right)+\tilde{e}_{l p}(t)
$$

where $x_{l p}$ denotes a complex quantity representing the scattering coefficient of the target between the lth subchannel and $p$ path; $\gamma=1+\beta$ represents the stretching in time of the reflected signal and $\beta=\frac{2\langle v, u\rangle}{c}$ is the
Doppler spreading factor, $u$ is direction-of-arrival (DOA) unit-vector and $c$ is the speed of propagation. $\tau_{p}$ is the roundtrip delay according along the $l$ th subchannel and $p$ path. The complex envelope of the received signal consists of the direct path and specular paths corresponding to a specific range cell containing the target can be described as

$$
\tilde{r}_{l}(t)=\sum_{p=0}^{P-1} a_{l} x_{l p} \phi_{l p}(n)+\tilde{e}_{l}(t)
$$

where

$$
\phi_{l p}(n)=e^{-j 2 \pi f_{l} \tau_{p}} e^{j 2 \pi f_{l} \beta_{p} n T_{P R I}}
$$

We can rewrite 15 as a column vector of dimension $L \times 1$ and we have

$$
y_{\mathrm{OFDM}}(n)=A X \phi(v, n)+e(n) \quad n=0,1, \ldots, N-1
$$

where

- $y_{\mathrm{OFDM}}(n)=\left[y_{0_{\mathrm{OFDM}}}(n), y_{1_{\mathrm{OFDM}}}(n), \ldots y_{L-1_{\mathrm{OFDM}}}(n)\right]^{T}$;

- $A=\operatorname{diag}(a)$ is an $L \times L$ complex diagonal matrix includes the transmitted subcarrier weights;

- $X=\operatorname{blkdiag}\left(x_{0}^{T}, x_{1}^{T}, \ldots, x_{L-1}^{T}\right)$ is an $L \times L P$ matrix; $x_{l}=x_{l} . \rho$ where $\rho=\left[\rho_{0}, \rho_{1}, \ldots, \rho_{P-1}\right]^{T}$ denotes the reflection coefficients over different paths. The number of possible propagation paths $\mathrm{P}$ adopted in our scenario are three, namely, direct-direct path $\left(\rho_{p}=1\right)$, direct-reflected path $\left(\rho_{p}=\rho e^{j \phi}\right)$, and reflect-reflect path $\left(\rho_{p}=\left(\rho e^{j \phi}\right)^{2}\right)$.

- $\phi(v, n)$ is an $L \times 1$ vector comprising the Doppler information of the target at the lth subchannel through the parameter $v$;

- $e(n)=\left[e_{0}(n), e_{1}(n), \ldots, e_{L-1}(n)\right]^{T}$ is an $L \times 1$ vector of measurement noise;

Then, combining the data from $N$ successive pulses columnwise into an $L \times N$ matrix, the OFDM measurement model is derived as follows:

$$
Y_{\mathrm{OFDM}}=A X \Phi+E
$$

where

- $Y_{\mathrm{OFDM}}=\left[y_{\mathrm{OFDM}}(0), y_{\mathrm{OFDM}}(1), \ldots y_{\mathrm{OFDM}}(N-1)\right]$;

- $\Phi^{t}=[\Phi(0), \Phi(1), \ldots, \Phi(N-1)]$ is an $L \times N$ matrix comprising the Doppler information of the target;

- $E=[e(0), e(1), \ldots, e(N-1)]$ is an $L \times N$ is a complex matrix that consists of measurement noise;

We construct a decision problem to choose between two possible hypotheses. We also consider noise and clutter as parts of the whole interference in this paper. Assume the target statistics are known while the interference statistics are not known. This corresponds, for example, to the situation where the radar is investigating for specific targets 
with identified signatures. Noise is assumed to be white with zero-mean complex Gaussian distribution. Besides, we suppose that the clutter reflection has been modeled by a collection of scatterers and is considered to be a zero-mean complex WSS Gaussian random process with unknown positive definite covariance matrix $\Sigma_{c}$ as

$$
X^{c} \phi^{c} \sim \operatorname{CN}_{L}\left(0, \Sigma_{c}\right)
$$

The clutter reflections are assumed to be temporally independent from pulse to pulse and vary with an unknown distribution from scan to scan. Therefore, we can rewrite (18) as

$$
Y_{\mathrm{OFDM}}=A \tilde{\Phi}^{t}+\tilde{E}
$$

where

$$
\begin{aligned}
& \tilde{\Phi}^{t}=X^{t} \Phi^{t} \\
& \tilde{E}=A X^{c} \Phi^{c}+E
\end{aligned}
$$

Based on these assumptions, the OFDM received measurements are distributed as

$$
Y_{\mathrm{OFDM}} \sim C \mathcal{N}_{L, N}\left(0, A R_{\tilde{\Phi}} A^{H}+R_{\tilde{E}}\right)
$$

where $R_{\tilde{\Phi}}$ is the target covariance matrix and $R_{\tilde{E}}$ is the interference covariance matrix which is given by [36]

$$
R_{\tilde{E}}=I_{N} \otimes\left(A \Sigma_{c} A^{H}+\sigma^{2} I_{L}\right)
$$

The target recognition problem in radar is formulated by (24), which is considered as a binary hypothesis testing problem.

$$
\left\{\begin{array}{l}
H_{0}: Y_{O F D M}=\tilde{E}, \text { No Target } \\
H_{1}: Y_{O F D M}=A \tilde{\Phi}^{t}+\tilde{E}, \text { Target } \\
\text { Exists }
\end{array}\right.
$$

We use the generalized likelihood ratio test (GLRT) detector which, had been derived in [33] for the considered hypothesis testing problem.

\section{Proposed method}

In this section, we present a recursive low-angle target tracking method to mitigate multipath nulls. We show that using frequency diversity of OFDM waveforms, and we can fill multipath nulls.

\subsection{Waveform optimization procedure}

In this section, an adaptive waveform design approach has been presented for designing the spectrum of the OFDM signal so that its complex weights match with the target and interference PSD. The maximization of the mutual information between unknown target impulse response and the received echoes as a function of the transmitted signal. This criterion is applied in this paper to design the complex weights of the transmitted signal and consequently improve the target tracking in low grazing angle scenarios.
If the transmitted waveform weights matrix $A$ in (18) is known, the conditional mutual information between $Y$ and $\tilde{\Phi}$ was given by [34]:

$$
\begin{aligned}
& \operatorname{MI}\left(Y_{\text {OFDM }} ; \tilde{\Phi} \mid A\right)=h\left(Y_{\text {OFDM }} \mid A\right)-h\left(\left(Y_{\text {OFDM }} \mid \tilde{\Phi}, A\right)\right) \\
& =N\left[\log \operatorname{det}\left(A R_{\tilde{\Phi}} A^{H}+R_{\tilde{E}}\right)-\log \operatorname{det}\left(R_{\tilde{E}}\right)\right]
\end{aligned}
$$

where $M I(x ; y)$ descibes the mutual information between $x$ and $y, h(x)$ represents the entropy of $x$ and $R_{x}=$ $E\left[x x^{H}\right]$ is the covariance matrix. We consider the power constraint to make the optimization problem nontrivial and consequently the optimization problem is stated as follows:

$$
\begin{array}{r}
A_{\mathrm{opt}}=\underset{A}{\arg \max }\left(N\left[\operatorname{logdet}\left(A R_{\tilde{\Phi}} A^{H}+\sigma^{2} I_{L \times L}\right)\right]\right) \\
\text { s.t } A A^{H} \leq P_{t}
\end{array}
$$

where $P_{t}$ is the total transmitted power in $d B W$. In this paper, we applied our waveform design approach [36] which the transmitted OFDM signal weights were optimized based on maximizing mutual information criterion. The optimized weights of the OFDM signal was driven as

$$
\beta_{l}=\max \left(-\frac{1}{\lambda}-\frac{\sigma_{u, l}^{2}}{(N+1) \sigma_{x, l}^{2}}, 0\right)
$$

where the parameter $\lambda$ can be calculated from (28).

$$
\sum_{l=1}^{L} \max \left(-\frac{1}{\lambda}-\frac{\sigma_{u, l}^{2}}{(N+1) \sigma_{x, l}^{2}}, 0\right)=P_{t}
$$

Figure 2 shows the flowchart of the proposed OFDM signaling scheme for low grazing angle target tracking scenarios. We will show that utilizing the optimized weights of the OFDM signal and using the proposed tracking algorithm, a more uniform detection coverage obtain in the presence of multipath propagation.

\subsection{Amplitude monopulse target tracking method}

We use a closed-loop amplitude monopulse target tracking method that utilizes the frequency diversity of OFDM signals to fill in multipath nulls. The target elevation angle by the amplitude monopulse method can be estimated as

$$
\hat{\theta_{d}}=\frac{1}{k_{m}} \operatorname{Re}\left(\frac{\Delta}{\Sigma}\right)
$$

where $k_{m}$ is the monopulse slope constant. It was shown that when the phase difference between the direct signal and reflected signal are in phase or out of phase will lead to destructive interference and consequently severe angle error estimation in the monopulse method.

The path length difference between the direct and reflected paths is a function of radar antenna height and target range and altitude. For a fixed set of these parameters, the amount of constructive or destructive interference is a function of RF carrier frequency. Operational 


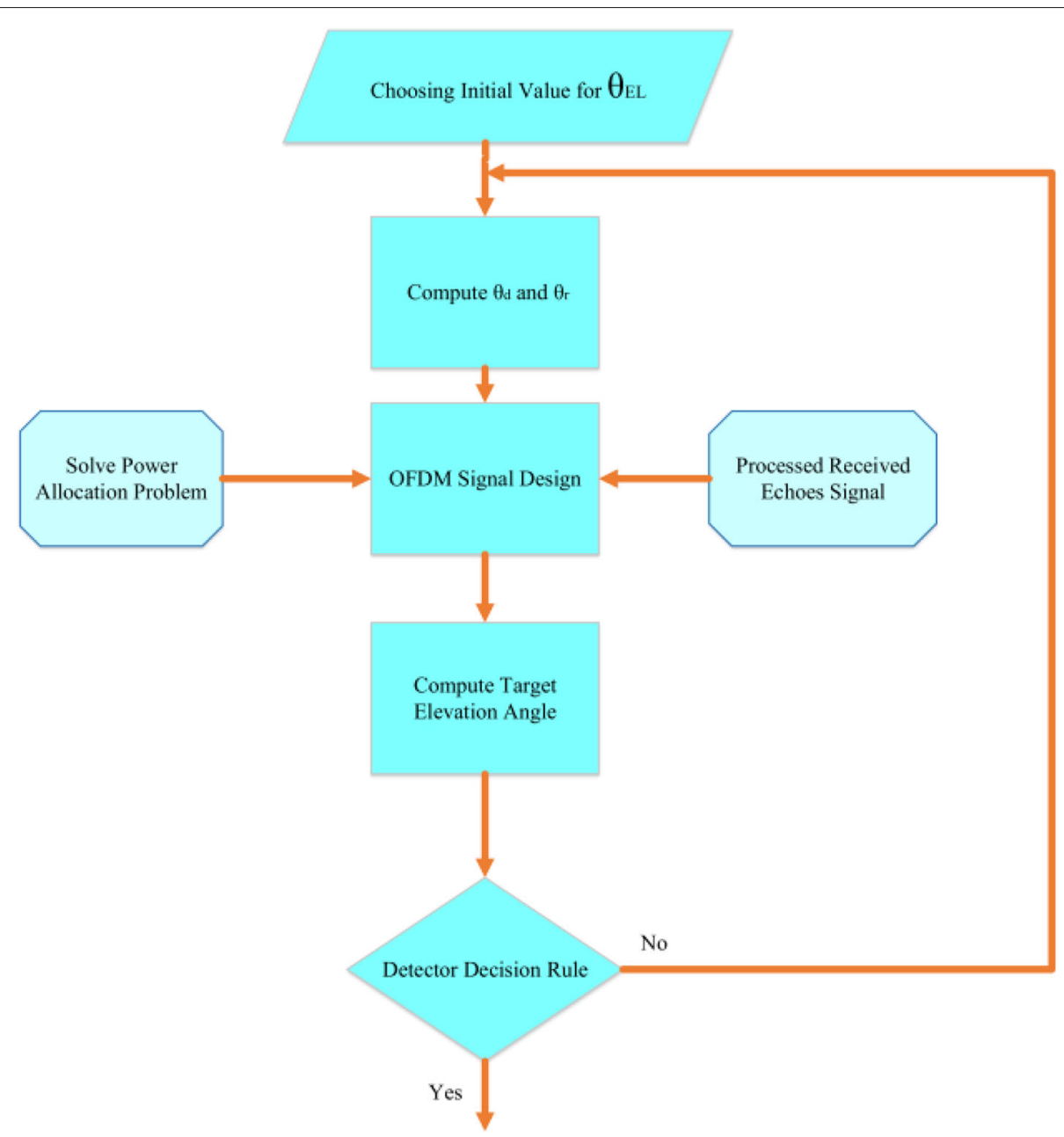

Fig. 2 Flowchart of the proposed OFDM signaling scheme for low grazing angle target tracking

radars attempt to mitigate nulls by transmitting a different RF carrier frequency from scan to scan.

It was shown in [20] that a sufficient change in the frequency will lead to decorrelate the phase measurement and make the measurement independent. With this assumption, the frequency spacing should be equal to

$$
\Delta f(H z)=\frac{c(m / s)}{2 D(m)}
$$

where $D$ is the depth of the target. In this paper, we propose a narrowband OFDM signal that by transmitting multiple, parallel RF subcarriers simultaneously in a single pulse succeeds in filling multipath nulls. In the simulations, we have assumed that $D=15 \mathrm{~m}$ and $\Delta f=10 \mathrm{MHz}$ will be obtained. On the other hand, the distance between the sub-carriers is the same, so it can result in frequency autonomy and frequency diversion to eliminate the multipath nulls. By transmitting a multicarrier OFDM signal with a bandwidth of a least that given by (30) should ensure that some of the subcarriers experience constructive interference or a peak while some may experience a null.

The proposed procedure for low-angle target tracking under multipath scenarios is indicated in Algorithm 1.

\section{Simulation results and discussions}

In this section, we consider several numerical examples to illustrate the performance of the proposed low grazing angle target approach. First, a description of the simulation setup is provided. The stochastic extended target involves a large number of scatterer points that are considered to move toward the radar with constant velocity and height, and its range varies from $8 \mathrm{~km}$ to $30 \mathrm{~km}$. The scattering coefficients of the target (i.e., the entries of $X$ ) are taken from a Gaussian ensemble with known $\operatorname{PSD}\left(C \mathcal{N}\left(0, C_{X}\right)\right)$. Then the scattering coefficients will be scaled to satisfy the required SNR. In each iteration, 1000 runs of the Monte Carlo simulation are performed, and the root mean square error (RMSE) of the estimated 


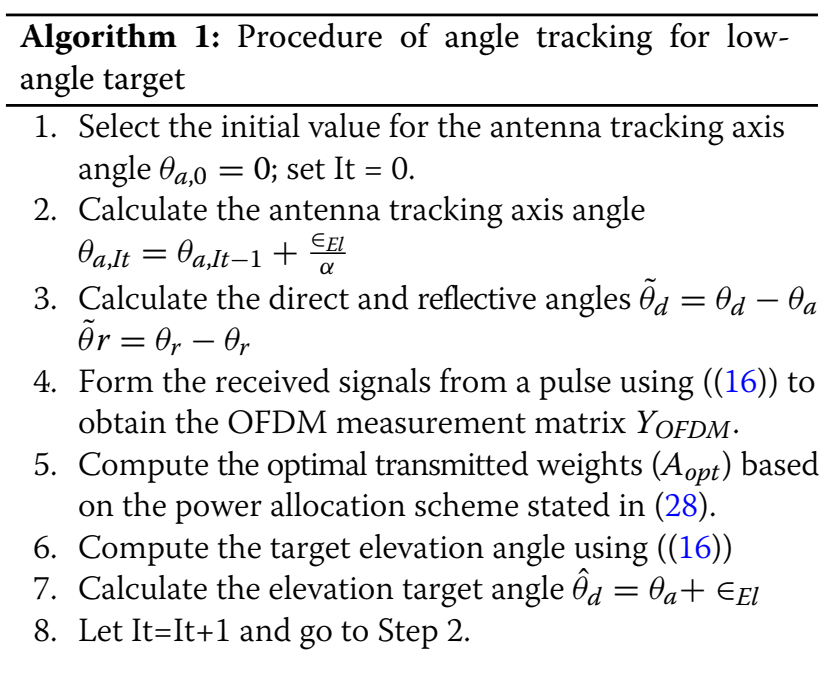

target elevation angle is computed at every 500 meter intervals.

Simulation Parameters for radar and environment are chosen as follow:

- Carrier frequency $f c=3 G H z$.

- Pulse repetition interval $T_{P R I}=1 \mathrm{~ms}$

- Pulse width $T=0.1 \mu \mathrm{s}$

- Subcarrier spacing $\Delta f=10 \mathrm{MHz}$

- Available bandwidth $B=170 \mathrm{MHz}$

- Number of coherent pulses with a given CPI $N=20$

- Number of subcarriers $L=16$

- Total transmitted power $P_{t}=10 \mathrm{dBW}$

- Number of multipaths $P=3$

- Antenna height $h_{a}=60$

- Target height $h_{t}=350$

- Sea RMS wave height $\sigma_{h}=0.07$

- Temperature $T=20 C$

- Static Dielectric Constant $\epsilon_{S}=69.08$

- Relaxation Time $\tau_{r}=9.15 \times 10^{-12}$

- Ionic Conductivity $\sigma_{i}=4.70 \times 10^{10}$

We present a recursive low-angle target tracking method using OFDM waveforms. The various simultaneous subcarrier frequencies will ensure that at least several subcarriers will always be outside multipath nulls on a given detection attempt to provide near-constant target visibility. We evaluate the performance of the proposed tracking algorithm via several simulations in the white Gaussian noise condition. The estimation performance is examined over 1000 Monte Carlo trials, and the RMSE of the estimated AOAs can be given at different target distances.

The sum and delta pattern simulated for the proposed amplitude monopulse method are shown in Fig. 3. Figure 4

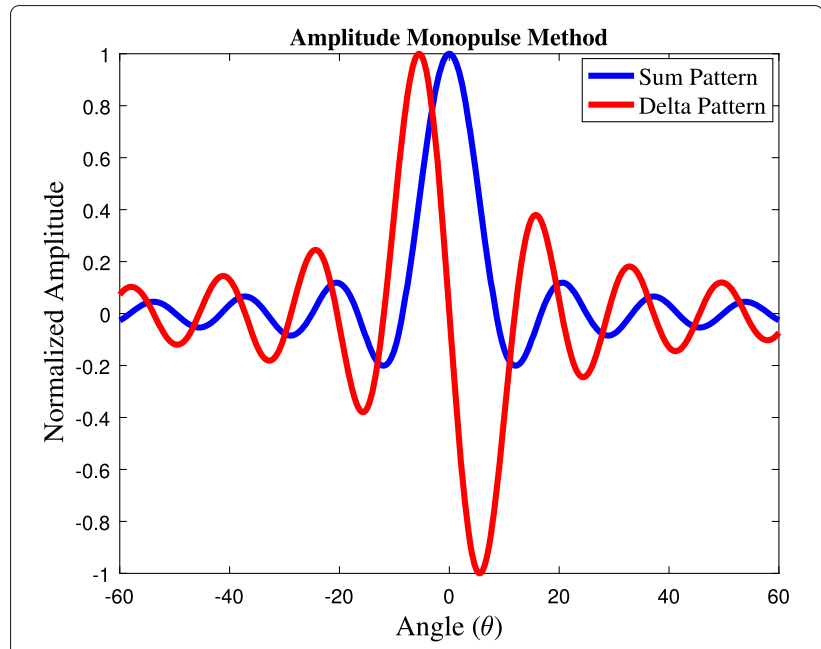

Fig. 3 Sum and delta pattern

shows the monopulse ratio which determines the target elevation angle based on (29).

Figure 5 shows the different power allocation schemes applied in the proposed low grazing angle target tracking algorithm. These power allocation schemes include single-carrier waveform [22], conventional equal-power multi-carrier waveform [35], the GLR-based multi-carrier waveform [12], and the proposed MI-based power allocation multi-carrier waveform.

Figure 6 shows the propagation coefficient in each sub-carrier for a single-carrier and a multi-carrier waveform. As can be seen in this figure, at some tracking distances, the amplitude for the single-carrier waveform is close to 0 , which is leading to a lower signal level and consequently, the more target estimation

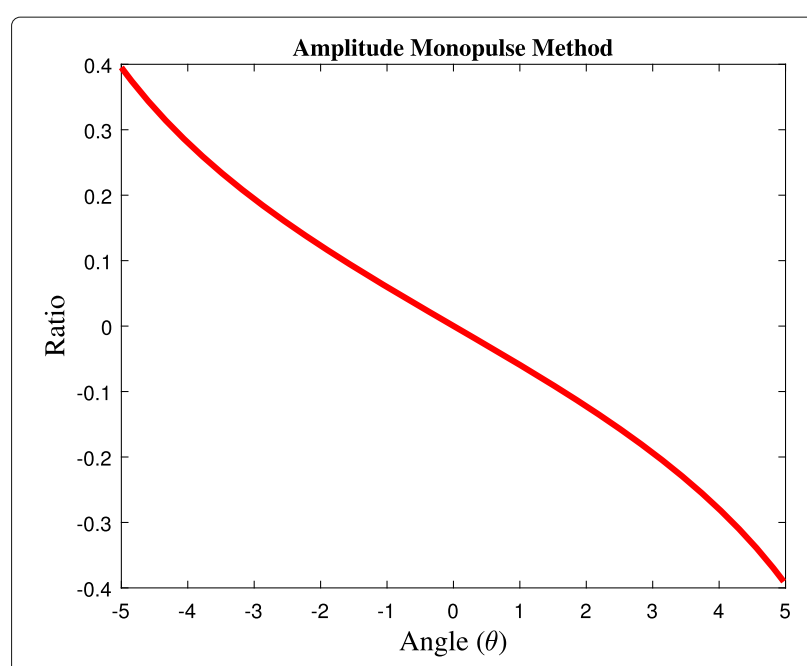

Fig. 4 Amplitude monopulse ratio 

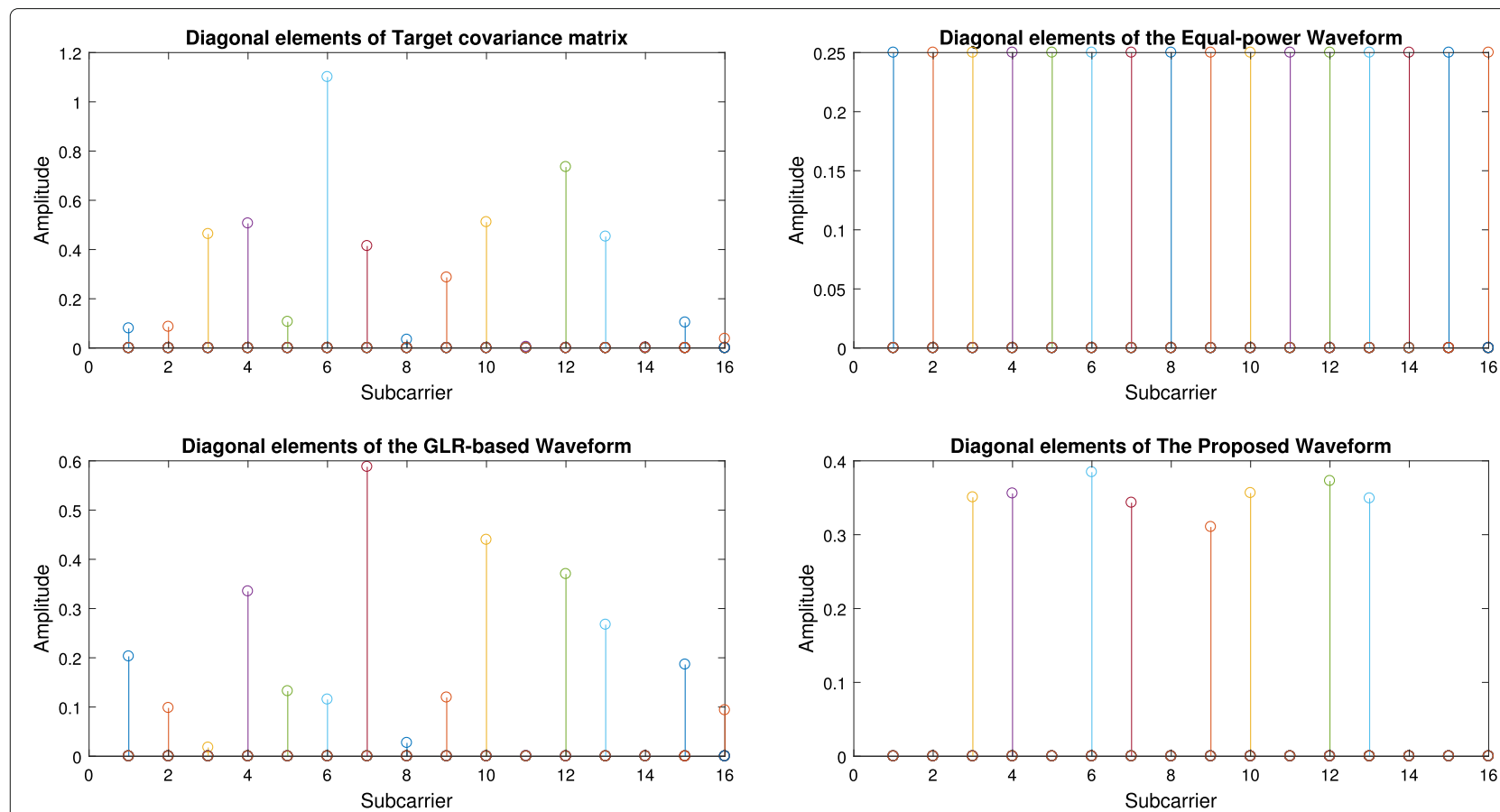

Fig. 5 Illustration of power allocation for different multicarrier waveforms. a Diagonal elements of target covariance matrix. b Diagonal elements of equal power waveform. c Diagonal elements of GLR-based waveform. d Diagonal elements of the proposed waveform $\left(L=16, P_{t}=10 \mathrm{dBW}\right)$

angle error. However, for distances that the propagation coefficient of the single-carrier waveform is close to 0 , we have appropriate propagation coefficient amplitudes and it means that we have sufficient energies in some carriers. Therefore, the target angle estimation will be improved using a multicarrier phase-coded (MCPC) waveform.

Figure 7 shows the RMSE curve of different approaches at different ranges. As shown in the figure, for the singlecarrier waveform (red color), target return at the radar receiver due to different phase differences indicates the peaks (maxima) and nulls (minima) corresponding to complete the constructive and destructive effect. While using multicarrier waveforms, the destructive effect will be removed, and the proposed approach can track the target with reasonable angle measurement error. As indicated in this figure, the best performance for the MCPC waveforms is achieved by using the proposed MI-based approach compared to other MCPC waveform design approaches. Furthermore, in Table 1, we have summarized

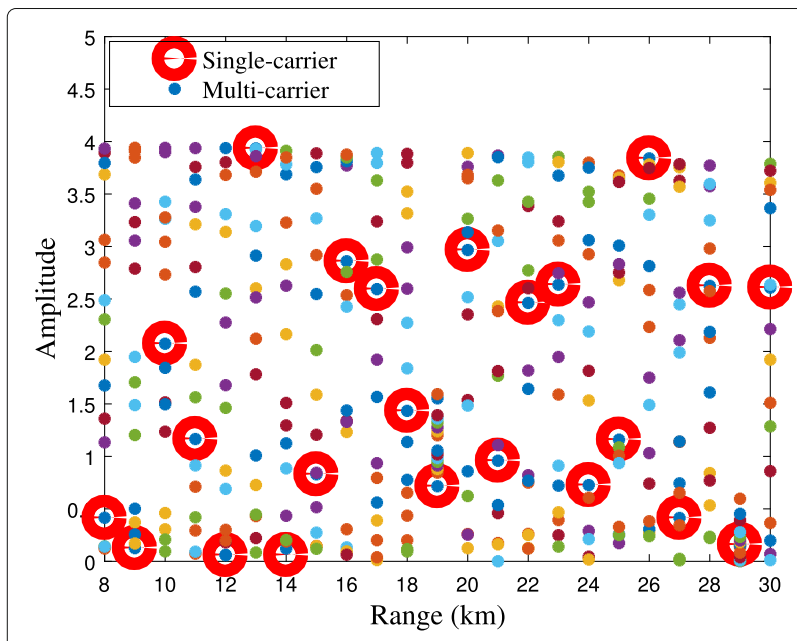

Fig. 6 Pattern propagation factor for single and MCPC waveform

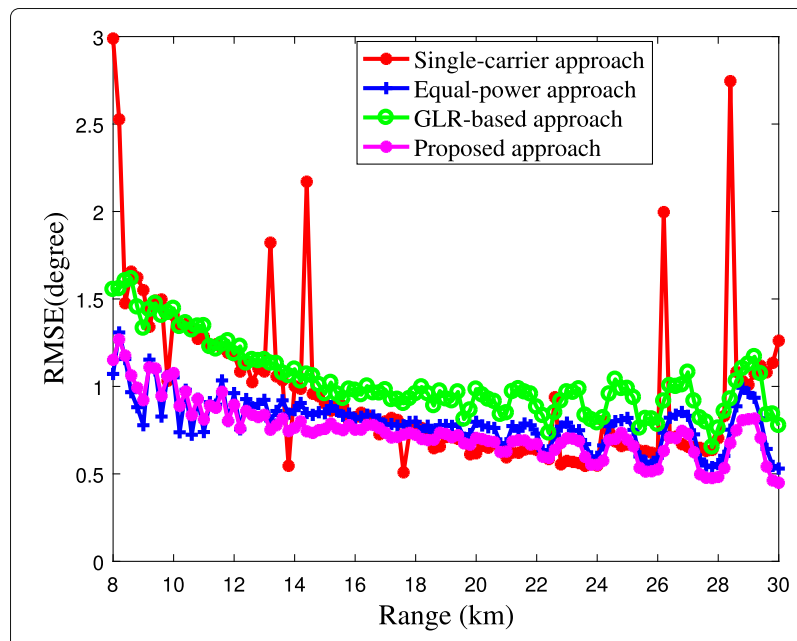

Fig. 7 RMSE versus range 
Table 1 Performance comparison of different approaches in low grazing angle target tracking scenario

\begin{tabular}{lllll}
\hline Approach & $\begin{array}{l}\text { Detection } \\
\text { probability }\end{array}$ & $\begin{array}{l}\text { Frequency } \\
\text { diversity }\end{array}$ & $\begin{array}{l}\text { Complexity } \\
\text { order }\end{array}$ & RMSE \\
\hline Single-carrier [22] & Average & Low & Low & High \\
Equal-power [35] & High & High & Low & Low \\
GLR-based [12] & High & High & Average & Average \\
The proposed & High & High & Average & Low \\
\hline
\end{tabular}

the comparison performance of different approaches in low grazing angle target tracking scenario.

Operational radars attempt to mitigate nulls by transmitting a different RF carrier frequency from scan to scan. This solution comes at the expense of the potential increase in time to detect a target. This paper addresses the use of an OFDM radar waveform to provide a more uniform detection coverage in the presence of multipath propagation by transmitting multiple, parallel RF subcarriers simultaneously in a single pulse to fill in the nulls. Simulation results justify this issue and show the importance of adaptive OFDM waveform design in low gazing angle target tracking scenarios.

\section{Conclusion}

In this paper, we addressed an edge computing problem of target tracking in a low grazing angle with OFDM radar. First, to make the propagation model more accurate, we incorporated realistic physical and statistical effects. Then, based on the characteristic of the OFDM radar system and the multipath propagation model, we developed an OFDM measurement model and derived a GLRT detector. And then, an adaptive design algorithm was proposed based on maximizing mutual information to enhance the detection performance to design the complex weights of the transmitted waveform. And finally, we showed that the detection performance is improved due to the multipath utilization as well as the adaptive design method. We also demonstrated that the detection process of multicarrier radar waveforms outperforms the singlecarrier OFDM radar waveform. Simulation results showed that by using the proposed OFDM waveform, the target tracking performance had been significantly improved in low grazing angle scenarios. In the future, we will explore other different criteria, e.g., ambiguity function, minimum mean square error, etc., to optimally design the transmit waveform to improve the tracking accuracy.

\footnotetext{
Abbreviations

DOA: Direction-of-arrival; GLRT: Generalized likelihood ratio test; LOC: Law of cosine High definition television; MCPC: Multicarrier phase-coded; MI: Mutual information; MIMO: Multiple input multiple output; OFDM: Orthogonal frequency division multiplexing; RMSE: Root mean square error
}

\section{Acknowledgments}

Not applicable.

\section{Authors' contributions}

VK and RM have completed the first draft of this paper. VK and RM participated in mathematical design of the proposed method and its computer implementation. VK and MK coordinated industrial application and raw data preparation, and helped out for study. All authors have read and approved the manuscript.

\section{Authors' information}

Not applicable.

\section{Funding}

Not applicable.

\section{Availability of data and materials}

All the data and computer programs are available.

\section{Competing interests}

The authors declare that they have no competing interests.

Received: 9 October 2019 Accepted: 31 December 2019

Published online: 13 January 2020

\section{References}

1. A. Drosopoulos, S. Haykin, Angle-of-arrival estimation in presence of multipath. Electron. Lett. 27(24), 2273-2274 (1991)

2. A. Karimian, C. Yardim, P. Gerstoft, W. S. Hodgkiss, A. E. Barrios, Multiple grazing angle sea clutter modeling. IEEE Trans. Antennas Propag. 60(9), 4408-4417 (2012)

3. H. Darvishi, M. A. Sebt, Adaptive hybrid method for low-angle target tracking in multipath. IET Radar Sonar Navig. 12(9), 931-937 (2018)

4. S. L. Silon, B. D. Carlson, Radar detection in multipath. IEE Proc. Radar Sonar Navig. 146(1), 45-54 (1999)

5. J. H. Zhao, J. Y. Yang, in 2006 CIE International Conference on Radar. Frequency diversity to low-angle detecting using a highly deterministic multipath signal model (IEEE, 2006), pp. 1-5. https://doi.org/10.1109/icr. 2006.343202

6. S. Kafshgari, R. Mohseni, Fluctuating target detection in presence of non Gaussian clutter in OFDM radars. AEU-Int. J. Electron. Commun. 67(10), 885-893 (2013)

7. S. Nazir, V. Stankovic, H. Attar, L. Stankovic, S. Cheng, Relay-assisted rateless layered multiple description video delivery. IEEE J. Sel. Areas Commun. 31(8), 1629-1637 (2012)

8. H. Attar, L. Stankovic, V. Stankovic, Cooperative network-coding system for wireless sensor networks. IET Commun. 6(3), 344-352 (2012)

9. H. Attar, D. Vukobratovic, L. Stankovic, V. Stankovic, in 2011 th IFIP International Conference on New Technologies, Mobility and Security. Performance analysis of node cooperation with network coding in wireless sensor networks (IEEE, 2011), pp. 1-4. https://doi.org/10.1109/ ntms.2011.5721048

10. V. Karimi, R. Mohseni, Y. Norouzi, M. J. Dehghani, Waveform design for cognitive radar with deterministic extended targets in the presence of clutter. Int. J. Commun. Netw. Syst. Sci. 9(06), 250 (2016)

11. V. Karimi, Y. Norouzi, in Proceedings of the Electronics New Zealand Conference (ENZCon'13). Target detection enhancement based on waveform design in cognitive radar, (New Zealand, 2013), pp. 40-45

12. S. Sen, A. Nehorai, Adaptive OFDM radar for target detection in multipath scenarios. IEEE Trans. Sig. Proc. 59(1), 78-90 (2010)

13. W. Du, R. L. Kirlin, Improved spatial smoothing techniques for DOA estimation of coherent signals. IEEE Trans. Sig. Proc. 39(5), 1208-1210 (1991)

14. J. Dai, Z. Ye, Spatial smoothing for direction of arrival estimation of coherent signals in the presence of unknown mutual coupling. IET Sig. Proc. 5(4), 418-425 (2011)

15. J. Ding, H. W. Chen, H. Wang, X. Li, Z. Zhuang, Low-grazing angle target detection and system configuration of MIMO radar. Prog. Electromagn. Res. 48, 23-42 (2013)

16. J. Ding, H. Chen, H. Wang, X. Li, Z. Zhuang, Low-grazing angle detection in compound-gaussian clutter with hybrid MIMO radar. Int. J. Antennas Propag. 2013 (2013). https://doi.org/10.1109/icosp.2014.7015355

17. Y. Xia, Z. Song, Z. Lu, Q. Fu, Target detection in low grazing angle with OFDM MIMO radar. Prog. Electromagn. Res. 46, 101-112 (2016) 
18. G. E. A. Franken, H. Nikookar, P. V. Genderen, in Proc. 3rd Eur. Radar Conf. Doppler tolerance of OFDM-coded radar signals, (Manchester, 2006), pp. 108-111

19. J. H. Wen, S. H. Lee, Y. F. Huang, H. L. Hung, A suboptimal PTS algorithm based on particle swarm optimization technique for PAPR reduction in OFDM systems. EURASIP J. Wirel. Commun. Netw. 2008, 14 (2008)

20. J.P. Stralka, Applications of orthogonal frequency-division multiplexing (OFDM) to radar, Ph.D. dissertation. (The Johns Hopkins Univ., Baltimore, 2008)

21. B. Gui, L. J. Cimini, Bit loading algorithms for cooperative OFDM systems. EURASIP J. Wirel. Commun. Netw. 2008(1), 476797 (2007)

22. S. C. Thompson, A. U. Ahmed, J. G. Proakis, J. R. Zeidler, M. J. Geile, Constant envelope OFDM. IEEE Trans. Commun. 56(8), 1300-1312 (2008)

23. P. Banelli, Theoretical analysis and performance of OFDM signals in nonlinear fading channels. IEEE Trans. Wirel. Commun. 2(2), 284-293 (2003)

24. V. Karimi, R. Mohseni, in Proceedings of the International Conference on Parallel and Distributed Processing Techniques and Applications (PDPTA'18). "Radar waveform design based on OFDM signals for cognitive radar application, (Las Vegas, 2018), pp. 168-169

25. V. Karimi, R. Mohseni, Intelligent target spectrum estimation based on OFDM signals for cognitive radar applications. J. Intell. Fuzzy Syst. 36(3), 2557-2569 (2019)

26. Z. Wang, A. Sinha, P. Willett, Y. Bar-Shalom, Angle estimation for two unresolved targets with monopulse radar. IEEE Trans. Aerosp. Electron. Syst. 40(3), 998-1019 (2004)

27. K. B. Yu, P. G. Kaup, C.orp. Lockheed Martin, Method and system for monopulse radar target angle determination (2010). U.S. Patent 7,859,451

28. S. Qiu, X. Ma, W. Sheng, Y. Han, R. Zhang, Robust adaptive monopulse algorithm based on main lobe constraints and subspace tracking EURASIP J. Adv. Sig. Proc. 2017(1), 14 (2017)

29. Y. X. Zhang, Q. F. Liu, R. J. Hong, P. P. Pan, Z. M. Deng, A novel monopulse angle estimation method for wideband LFM radars. Sensors. 16(6), 817 (2016)

30. S. L. Wang, Z. H. Xu, X. Liu, W. Dong, G. Wang, Subarray-based frequency diverse array for target range-angle localization with monopulse processing. IEEE Sensors J. 18(14), 5937-5947 (2018)

31. X. Shen, R. Chang, D. Yuan, Pseudo-monopulse tracking method for low profile mobile satellite antenna system. AEU-Int. J. Electron. Commun. 101, 160-167 (2019)

32. D. Park, E. Yang, S. Ahn, J. Chun, Adaptive beamforming for low-angle target tracking under multipath interference. IEEE Trans. Aerosp. Electron. Syst. 50(4), 2564-2577 (2014)

33. V. Karimi, R. Mohseni, S. Samadi, OFDM waveform design based on mutual information for cognitive radar applications. J. Supercomput. 75(5), 2518-2534 (2019)

34. M. R. Bell, Information theory and radar waveform design. IEEE Trans. Inf. Theory. 39(5), 1578-1597 (1993)

35. L. Litwin, M. Pugel, The principles of OFDM. RF Sig. Proc. 2, 30-48 (2001)

36. V. Karimi, R. Mohseni, S. Samadi, Adaptive OFDM Waveform Design for Cognitive Radar in Signal-Dependent Clutter. IEEE Syst. J. (2019). https:// doi.org/10.1109/jsyst.2019.2943809

\section{Publisher's Note}

Springer Nature remains neutral with regard to jurisdictional claims in published maps and institutional affiliations.

\section{Submit your manuscript to a SpringerOpen ${ }^{\circ}$ journal and benefit from:}

- Convenient online submission

- Rigorous peer review

- Open access: articles freely available online

- High visibility within the field

- Retaining the copyright to your article

Submit your next manuscript at $>$ springeropen.com 